\title{
Multi-colour detection of gravitational arcs
}

\author{
Matteo Maturi ${ }^{1}$, Sebastian Mizera ${ }^{2}$, and Gregor Seidel ${ }^{3}$ \\ ${ }^{1}$ Institut für Theoretische Astrophysik, Zentrum für Astronomie, Universität Heidelberg, Philosophenweg 12, \\ 69120 Heidelberg, Germany \\ e-mail: maturi@uni-heidelberg.de \\ 2 Girton College, University of Cambridge, Huntingdon Road, Cambridge CB3 0JG, UK \\ 3 Max-Planck-Institut für Astronomie, Königstuhl 17, 69117 Heidelberg, Germany
}

Received 3 April 2013 / Accepted 7 May 2014

\section{ABSTRACT}

\begin{abstract}
Strong gravitational lensing provides fundamental insights into the understanding of the dark matter distribution in massive galaxies, galaxy clusters, and the background cosmology. Despite their importance, few gravitational arcs have been discovered so far. The urge for more complete, large samples and unbiased methods of selecting candidates increases. Several methods for the automatic detection of arcs have been proposed in the literature, but large amounts of spurious detections retrieved by these methods force observers to visually inspect thousands of candidates per square degree to clean the samples. This approach is largely subjective and requires a huge amount of checking by eye, especially considering the actual and upcoming wide-field surveys, which will cover thousands of square degrees. In this paper we study the statistical properties of the colours of gravitational arcs detected in the 37 deg $^{2}$ of the CFHTLS-Archive-Research Survey (CARS). Most of them lie in a relatively small region of the $\left(g^{\prime}-r^{\prime}, r^{\prime}-i^{\prime}\right)$ colour-colour diagram. To explain this property, we provide a model that includes the lensing optical depth expected in a $\Lambda \mathrm{CDM}$ cosmology that, in combination with the sources' redshift distribution of a given survey, in our case CARS, peaks for sources at redshift $z \sim 1$. By furthermore modelling the colours derived from the spectral energy distribution of the galaxies that dominate the population at that redshift, the model reproduces the observed colours well. By taking advantage of the colour selection suggested by both data and model, we automatically detected 24 objects out of 90 detected by eye checking. Compared with the single-band arcfinder, this multiband filtering returns a sample complete to $83 \%$ and a contamination reduced by a factor of $\sim 6.5$. New gravitational arc candidates are also proposed.
\end{abstract}

Key words. dark matter - galaxies: clusters: general - methods: observational - gravitational lensing: strong

\section{Introduction}

Strong gravitational lensing is a powerful tool used to probe dark matter and in general to constrain cosmological models. The power is in its high non-linearity and sensitivity to mass distributions and the geometry of space-time (e.g. see reviews, Wambsganss 1998; Schneider et al. 1999; Kochanek 2006; Bartelmann 2010). It is variously applied, for instance in (1) studying high redshift objects whose strongly magnified image allows examining these objects in more detail, which would be very difficult otherwise (e.g. Kneib et al. 2004; Richard et al. 2008, 2011; Zitrin \& Broadhurst 2009; Bradač et al. 2012; Coe et al. 2013); (2) determining the Hubble constant via time delays (e.g. Coles 2008; Suyu et al. 2010; Tewes et al. 2013); (3) measuring of dark matter amount and distribution of the lenses, in particular of galaxy clusters (Broadhurst et al. 2005; Zitrin et al. 2009; Merten et al. 2009); and (4) studying intrinsic properties of dark matter, such as its collisional cross section, by investigating major merger events between galaxy clusters (Clowe et al. 2004; Merten et al. 2011). Moreover, giant arcs are a valuable tool for constraining cosmology, since their number is very sensitive to cosmological parameters and structure formation (Meneghetti et al. 2013). Of particular interest is the discrepancy between predicted and observed number in the sky of these arcs, as highlighted by (Bartelmann et al. 1995, 1998; Meneghetti et al. 2000; Dalal et al. 2004; Meneghetti et al. 2003), even if recent studies alleviated this discrepancy by introducing more accurate cluster models based on numerical $N$-body simulations (Meneghetti et al. 2000; Torri et al. 2004; Meneghetti et al. 2003; Li et al. 2005; Horesh et al. 2005) and halo models (Fedeli et al. 2006).

Many gravitational arcs have been found around large galaxies (see e.g. Bolton et al. 2006; Anguita et al. 2012). In particular, only few giant arcs caused by galaxy clusters are known. The search for them is mostly focused on bright X-ray cluster, because these are very efficient in producing strong-lensing features. This selection biases the actual sample, since these clusters tend to be non-relaxed, which limits the possibilities of this powerful observable. Up to now, only a few hundred of cases have been confirmed (see e.g. Fassnacht et al. 2004; Cabanac et al. 2007; Faure et al. 2008; Jackson 2008; Limousin et al. 2009; Verdugo et al. 2011; More et al. 2012; Bayliss et al. 2011; Oguri et al. 2012).

To solve these problems, automatic arc-detection methods have been proposed in the literature to produce unbiased samples, but they still suffer from strong contamination and require much human intervention checking by eye thousands of candidates per square degree. The need of much human time and the subjective outcome both limit the applicability of these procedures (see e.g. Cabanac et al. 2007; More et al. 2012). Recently, Joseph et al. (2014) proposed a promising principal component analysis based method that specialises in detecting arcs that are highly blended with galaxies, and Gavazzi et al. (2014) used two-band imaging to reduce the contamination of their sample. 
Our results were derived independently and provide additional insights in the form of the colour statistics of arcs by providing a model based on the spectral energy distribution (SED) of highredshift galaxies, the lensing optical depth, and the data depth. In addition, their work is focused on galaxy lensing and not on clusters and groups lensing.

We aim at enlarging the sample of gravitational arcs by using the arcfinder proposed by Seidel \& Bartelmann (2007) combined with a new colour-selection procedure discussed in this work. The method allows a strong reduction of the sample contamination, which alleviates the efforts devoted to the final validation of the most promising candidates. We process the CFHTLSArchive-Research Survey (CARS Erben et al. 2009) data, which cover 37 square degrees, to verify the efficiency of the method and to detect new gravitational arcs.

The paper is organised as follows: in Sects. 2 and 3 we introduce the basics of strong gravitational lensing and the data set characteristics, in Sect. 4 the arcfinder and its applications are described, while in Sect. 5 we characterise the colour properties of arcs to be used for the subsequent selection. In Sect. 6 the full method is discussed, and in Sect. 7 we present the final sample of arcs. Our conclusions are finally drawn in Sect. 8.

\section{Basics of strong gravitational lensing}

All lensing quantities derive from the gravitational potential, $\Phi$, of the matter placed along the line of sight on a thin plane placed between the observer and the background sources

$\psi(\boldsymbol{\theta}) \equiv \frac{2}{c^{2}} \frac{D_{\mathrm{ds}}}{D_{\mathrm{d}} D_{\mathrm{s}}} \int \Phi\left(D_{\mathrm{d}} \boldsymbol{\theta}, z\right) \mathrm{d} z$

Here, $\psi(\boldsymbol{\theta})$ is the so-called lensing potential, which depends on the angular position, $\boldsymbol{\theta}$, in the plane of the sky, $c$ is the speed of light, and $D_{\mathrm{ds}}, D_{\mathrm{d}}$ and $D_{\mathrm{s}}$ are the lens-source, the observer-lens and the observer-source angular-diameter distances, respectively.

Gravitational lensing maps the lens plane into the source plane via the lens equation

$\boldsymbol{\beta}=\boldsymbol{\theta}-\nabla \psi(\boldsymbol{\theta})$,

which can be linearised because sources such as distant galaxies are much smaller than the typical scale on which the lens properties vary. The induced image distortion is thus expressed by the Jacobian of the linearised lens equation

$A \equiv \frac{\partial \boldsymbol{\beta}}{\partial \boldsymbol{\theta}}=\left(\delta_{i j}-\frac{\partial^{2} \psi(\boldsymbol{\theta})}{\partial \theta_{i} \partial \theta_{j}}\right)=\left(\begin{array}{cc}1-\kappa-\gamma_{1} & -\gamma_{2} \\ -\gamma_{2} & 1-\kappa+\gamma_{1}\end{array}\right)$,

where $\kappa$ is the convergence and $\gamma=\gamma_{1}+\mathrm{i} \gamma_{2}$ is the complex shear. Since $A$ is symmetric, it can always be diagonalised, and its two real eigenvalues, $\lambda_{\mathrm{t}}=1-\kappa-\gamma$ and $\lambda_{r}=1-\kappa+\gamma$, represent the distortion of an infinitesimal source in tangential and radial directions relative to the lens centre, respectively. The lengthto-width ratio $(L / W$ hereafter) of the image is thus defined as $q=\lambda_{\mathrm{t}} / \lambda_{r}$ and its magnification factor reads

$\mu=\frac{1}{(1-\kappa)^{2}-\gamma_{1}^{2}-\gamma_{2}^{2}}$.

For more details on gravitational lensing, see for example Wambsganss (1998), Schneider et al. (1999), or Bartelmann (2010).
Table 1. Name, position (J2000), and area of the three fields covered by CARS.

\begin{tabular}{cccc}
\hline \hline Field & RA & Dec & Area $\left(\mathrm{deg}^{2}\right)$ \\
\hline$W 1$ & $02: 18: 00$ & $-07: 00: 00$ & 21 \\
$W 3$ & $14: 17: 54$ & $+54: 30: 31$ & 5 \\
$W 4$ & $22: 13: 18$ & $+01: 19: 00$ & 11 \\
\hline
\end{tabular}

\section{Data set}

To test our arcfinding method and possibly discover new strong-lensing features, we processed stacked images from the CFHTLS-Archive-Research Survey (CARS, Erben et al. 2009), a set of three high-galactic-latitude patches covering a total of $37 \mathrm{deg}^{2}$ and produced with the publicly available observations obtained with the MegaPrime camera mounted at the CanadaFrance-Hawaii Telescope (CFHT) within the Canada-FranceHawaii-Telescope Legacy Survey ${ }^{1}$. The MegaPrime is an optical camera with a mosaic of $9 \times 4$ CCDs of $2048 \times 4096$ pixels, each sampling 0.186 arcsec over a field of view of one square degree (see e.g. Boulade et al. 2003). All observations were obtained through the filters $u^{\star}, g^{\prime}, r^{\prime}, i^{\prime}$, and $z^{\prime}$. The three patches of sky covered by CARS are listed in Table 1 . The deep co-added images were produced using the GaBoDS/THELI pipeline on a pointing/colour basis after rejecting all exposures with a problematic CFHT quality assessment (Erben et al. 2005). All details about CARS can be found in Erben et al. (2009).

The depth of images ( $A B$ magnitude), defined as the $5 \sigma$ detection limit in a $2^{\prime \prime}$ radius aperture, is typically 25.24, 25.30, 24.36, 24.68, and 23.20 for the $u^{\star}, g^{\prime}, r^{\prime}, i^{\prime}$, and $z^{\prime}$ bands, respectively. The measured seeing for all co-added images is well below 1.0 arcsec in all bands, except for the W1p3p $3 u^{\star}$-band image, which has a seeing of 1.1 arcsec, and therefore was ignored in this work. The seeing quality is crucial for detecting arcs because of their very small width. A large seeing would strongly affect their signal-to-noise ratio and reduce $L / W$, which would make the detection significantly more difficult because this is their most distinctive signature.

In addition to the publicly available data, we used the $g^{\prime}$ and $r^{\prime}$ bands to produce a weighted co-addition for each field to obtain an image with a higher signal-to-noise ratio, over which we ran the arcfinder segmentation, as discuss below. In the coaddition, we ignored the $u^{\star}$ and $z^{\prime}$ bands because of their lower intrinsic depth, and the $i^{\prime}$ band because large elliptical galaxies are too prominent in it. Arcs tend to be close to such objects which would strongly affect their segmentation.

\section{Image segmentation}

As a first step of searching for gravitational arcs, we adopted the arcfinder described in Seidel \& Bartelmann (2007) to produce the image segmentation necessary to detect elongated objects, and obtain important geometrical information, such as their length, $L$, and length-to-width ratio, $L / W$. Both are fundamental quantities for a subsequent object selection. In this work we briefly summarise the arcfinder algorithm in the Appendix.

We calibrated the arcfinder parameters on an empirical basis by selecting cut-outs centred on arcs previously discovered in the CARS data, and by adjusting parameters so that arcs are recognised as such. At the same time, we controlled and evaluated the number of spurious detections to minimise their presence.

1 http://www.cfht.hawaii.edu/Science/CFHLS 


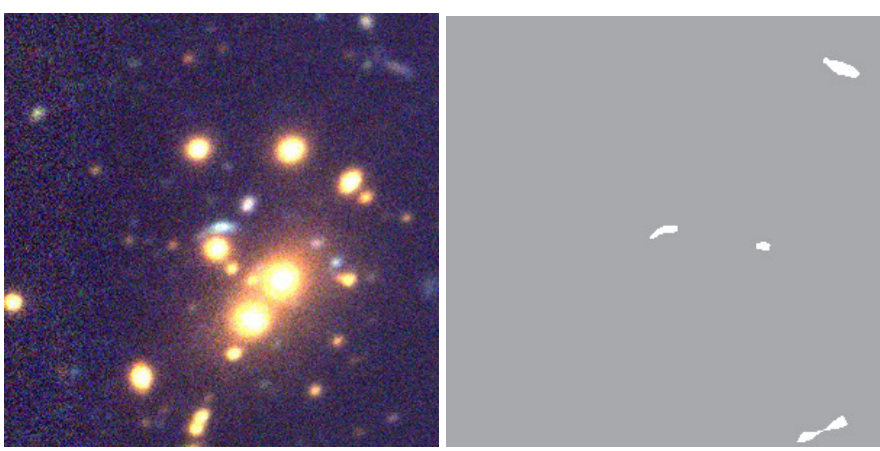

Fig. 1. One of the arcs detected by More et al. (2012) in the centre of the image used to initialise arcfinder parameters. The arc is clearly detected and there are very few false detections compared with all objects in the field, even at the segmentation stage before the selection process.

At this stage, we set the final segmentation parameters but defined only very relaxed geometrical constraints, that is, length and length-to-width ratio, for the very first selection of suitable sources. This is to obtain a wide overview of statistics of their geometrical properties to gain the necessary information for the actual calibration of the arcfinder, as discussed in the next paragraphs. As an additional rejection criterion we removed circular areas surrounding bright stars, which might create false detections because of their halos and diffraction spikes. The radius in pixels, $r=420 f^{1 / 3}$, of these areas is related to the stellar flux, $f$, as derived from the UCAC3 catalogue (Zacharias et al. 2010). The same catalogue was used to define their locations in the field of view. Moreover, we set an upper limit in the surface brightness of candidates, because arcs tend to be faint. This limit was set to $0.6 \mathrm{ADU} / \mathrm{s}$, and was empirically chosen given the surface brightnesses of the arcs used in the calibration. The most important parameters used for the segmentation are listed in Appendix A. These parameters might be refined by iterating the process of inclusion of new arcs discovered at each step. This might be strictly necessary if none or only few known arcs are present in the field under investigation. In our case we have a sufficiently large sample for a proper and robust calibration. In fact, with a unique set of parameters, we detected arcs with very different shapes, length-to-width ratios, curvature, and environment, ranging from cluster to galactic arcs.

In Fig. 1 we present a small portion of one of the cut-outs used for the calibration together with the related segmentation produced by the arcfinder. Clearly, only very few objects appear in the right panel of the figure. Detected objects include a giant arc in the middle, one object facing the observer edgeon, and an elongated feature resulting from blending of two objects. Blending of the lower right detection may appear excessive, given the large separation of the two objects, but we have to keep in mind that the segmentation is not based on a brightness cut-off criterion, as in the case of many other algorithms. Our segmentation algorithm measures coherent patterns in local second-brightness moments which, for these two objects, are aligned along a common direction which results in their merging as a single detection. We used an aggressive parameter for blending to avoid splitting of arcs with strong luminosity variations along their major axis.

With this segmentation and very loose filtering applied so far to the CARS data, and with very relaxed geometrical constraints used to acquire a wide statistical understanding of the data (the final selection still has to be made), we obtained an initial sample of 201699 sources, the number density of which across the survey is shown in Fig. 2. The clear fluctuations in number density follow the intrinsic variations of the object distribution and, more importantly, depend on the large variations of the image depth across the survey field. We account for these variations during the arcfinding process, where we perform a local estimate of the noise level to retrieve a sample with uniform signal-to-noise ratio properties. Note that this initial catalogue is largely dominated by spurious objects, since the most stringent constraints are not yet applied. In Fig. 3 we plot the probability distribution and cumulative function of the main geometrical properties, that is length and length-to-width ratio, of the entire sample of sources obtained with the arcfinder.

In this step, we aim at completeness by setting only weak constraints on the detection shapes we expect from strong lensing in combination with the point spread function (PSF hereafter) convolution, hence we chose a minimal length-to-width ratio of $L / W>4$ instead of bolder values, such as 7 or 10 , which are typical for well-resolved strong-lensing features. Lower values cannot be adopted, because they are typical for most of the sources in the field (e.g. galaxies, amongst others). The lack of an additional length constraint would allow for objects smaller than the image PSF. Because of this reason, we set a minimum length of $L>15$ pixels $(2.9$ arcsec $)$. These constraints are shown as green vertical lines in Fig. 3. With these selection parameters we reduced the sample size to 36026 objects, which yields an average of about 970 detections per square degree. This is the final sample as provided by the arcfinder when applied onto a single band. Even if the number of candidates seems large (and in fact it still contains many false detections), we have to keep in mind that we started with $7 \times 10^{6}$ objects detected with SExtractor within the survey (Bertin \& Arnouts 1996) and that we search for objects down to the noise limit. This shows how, with the arc segmentation alone, we already have an efficient and powerful filter, even if it is not sufficient to obtain a reliable automatic procedure. In the next section we show how expanding the method to include three bands improves the situation.

\section{Colour properties of the sample}

After obtaining the source segmentation, curvature, length and $L / W$, we obtain a sample of objects with the correct geometrical properties, meaning faint, thin, and elongated sources. This is still not enough for a clear distinction between gravitational arcs and other astrophysical sources or noise fluctuations, however in this work we address this problem with photometry, in particular of colours. We show, in fact, that the population of sources with the highest probability to experience strong lensing (thus resulting in gravitational arcs) is dominated by a fairly uniform population of galaxies at redshift $z \approx 1$. These objects are mainly small galaxies with large star-forming regions and therefore specific colour properties (Willmer et al. 2006).

\subsection{Theoretical motivation}

The probability of observing sources with strong-lensing features is given by their number-density distribution dependent on redshift,

$n(z)=\frac{z^{a}}{z^{b}+c z^{2}+d}$

where $a=1.79, b=0.17, c=8.62, d=0.03$, which is the redshift distribution proposed by Benjamin et al. (2007) with an 

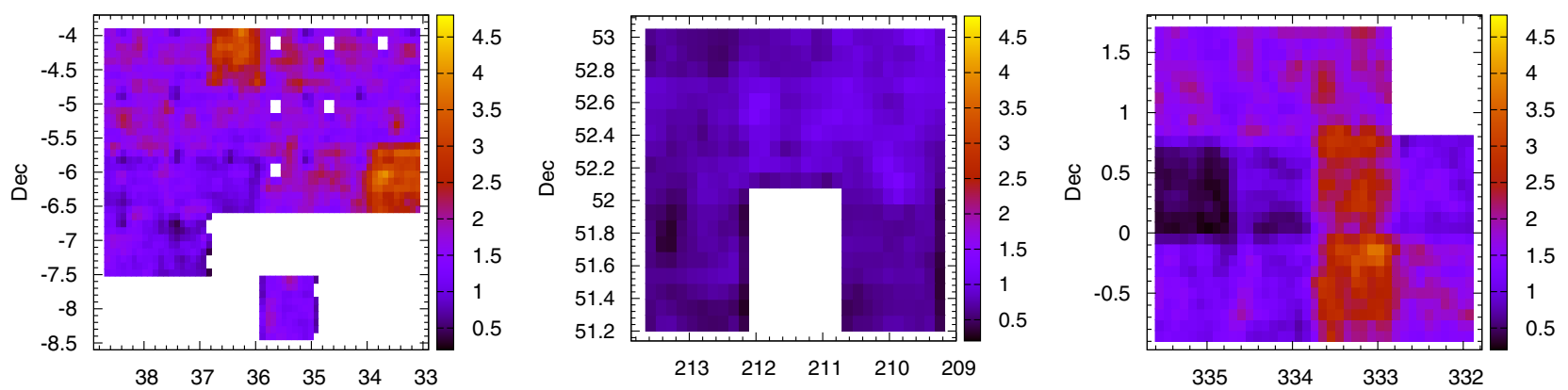

Fig. 2. Number density per $\operatorname{arcmin}^{2}$ of all objects identified by the arcfinder before applying geometrical and colour-selection criteria, which were applied in subsequent steps to identify the arc candidates. Since the more stringent constraints are not applied yet, these maps are dominated by spurious detections, which shows their distribution in the survey. The fluctuations in number density are expected because of the intrinsic inhomogeneous distribution of the sources and the large inhomogeneities of the image depth across the survey. We took this into account by measuring the noise locally. The three CARS fields, W1 (left), W3 (middle) and W4 (right), are shown.
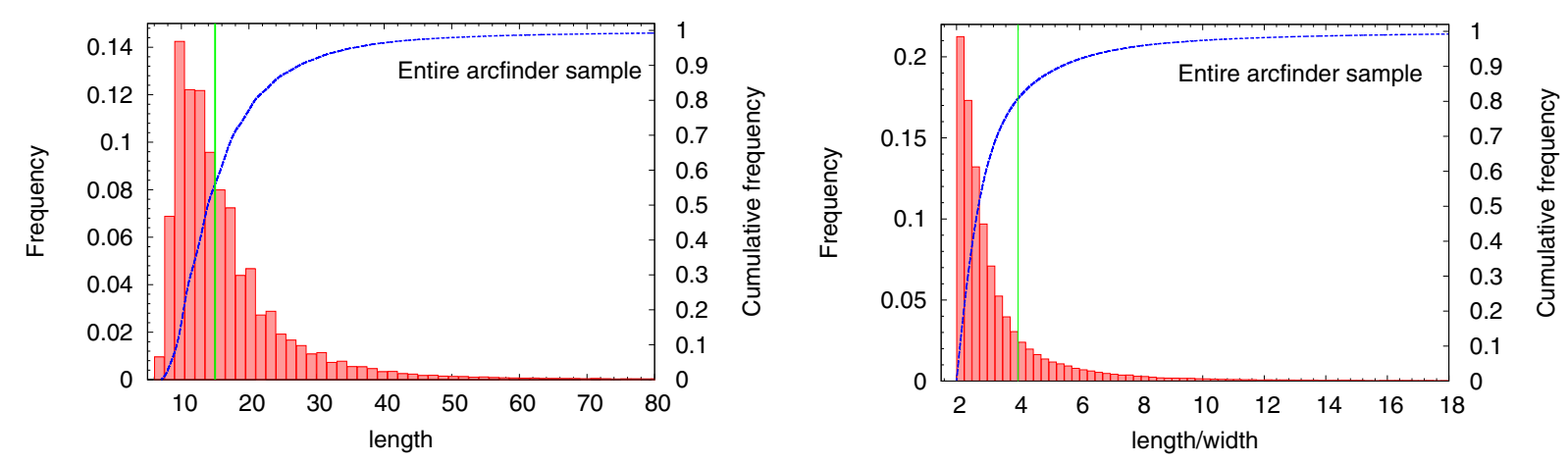

Fig. 3. Probability distribution (filled bars) and cumulative function (blue line) of the length, expressed in pixels of 0.186 arcsec in size, and the length-to-width ratio of all objects detected with the arcfinder before the application of the final geometrical and colour selection, in the left and right panels, respectively. The vertical green lines represent the respective lower limits adopted for the final candidate selection to maximize the completeness given the limits imposed by the data PSF.

additional term in the denominator, and the lensing cross-section of an intervening lens,

$\sigma_{\mathrm{d}}\left(z_{\mathrm{s}}\right)=\eta_{0}^{2} \int_{B_{1}} \frac{\mathrm{d}^{2} x}{|\mu(x)|}$

defined as the area on the source plane, where sources are imaged as arcs with an $L / W$ higher than a given minimum, $d$. Here $\mu$ is the lensing magnification as introduced in Eq. (4), $B_{1}$ represents the area in the lens plane for which the condition $L / W>d$ is met, $x=\theta / \theta_{0}$ are dimensionless coordinates scaled by $\theta_{0}$ and $\eta_{0}=\left(D_{\mathrm{s}} / D_{\mathrm{l}}\right) \theta_{0}$ in the lens and source plane, respectively. For simplicity we assumed point sources and spherically symmetric lenses modelled as NFW halos (Navarro et al. 1997) with scale radii $r_{\mathrm{s}}$. The redshift distribution of the number density of expected arcs, $\Gamma_{\mathrm{d}}\left(z_{\mathrm{s}}\right)=n\left(z_{\mathrm{s}}\right) \tau\left(z_{\mathrm{s}}\right)$, is obtained by multiplying the sources number density, $n(z)$, with the sum of the cross section of all lenses between the observer and the sources divided by the area of the source plane, that is the lensing optical depth,

$\tau_{\mathrm{d}}\left(z_{\mathrm{s}}\right)=\frac{1}{4 \pi D_{\mathrm{s}}^{2}} \int_{0}^{z_{\mathrm{s}}} \int_{0}^{\infty} N(m, z) \sigma_{\mathrm{d}}\left(m, z, z_{\mathrm{s}}\right) \mathrm{d} z \mathrm{~d} m$.

Here, $N(m, z) \mathrm{d} z$ is the total number of haloes with mass $m$ enclosed in the cosmic volume within redshifts $z$ and $z+\mathrm{d} z$ as defined by the differential mass function of Sheth $\&$ Tormen (2002). In the left and right panels of Fig. 4 we show the source distribution of the CARS galaxy sample together with the lensing optical depth, $\tau_{d}$, and the resulting distribution of the $\operatorname{arcs}, \Gamma_{d}$.

Here, we can safely use the redshift distribution of all sources in the survey, which is dominated by weakly lensed sources, and fully ignore their flux enhancement, which would be caused by a strong lens. This is because while lensing does increase the total flux of a source, it does not affect its surface brightness, which is what the initial arcfinder detection is more sensitive to. The peak of the number of strongly lensed sources at $z \approx 1$, is caused by the steep rise of the optical depth and the drop of the source number density with increasing redshift. The high-redshift tail of our model overestimates the actual number of expected arcs because the best fit of the sources overestimates the actual data, which drop for $z>1.3$ to become zero for $z>2$. For this reason, we can assume that the largest number of strongly lensed sources in our data are confined to a relatively narrow redshift interval around $z \approx 1$.

Many different works based on numerical $N$-body simulations and halo-models have been devoted to evaluating the lensing optical depth with extended sources, the lens intrinsic ellipticity, and the presence of substructures within large haloes (see e.g. Meneghetti et al. 2003; Li et al. 2005; Fedeli et al. 2006). These additional details mostly increase the efficiency of lenses to produce arcs and only marginally affect its redshift dependence, which we are interested in. With our simple model we just focus on the sources instead of on the lenses, in contrast to most of these studies. It is neither meant to produce a detailed prediction of the number of observable arcs nor to define 

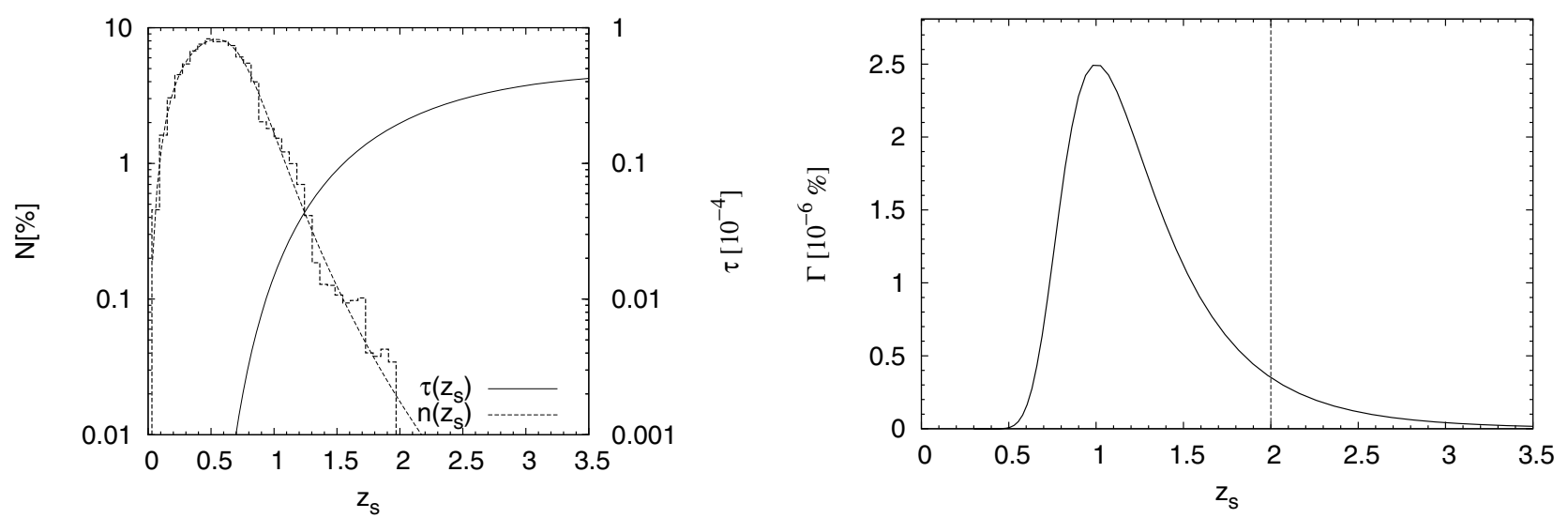

Fig. 4. Left panel: lensing optical depth, $\tau_{\mathrm{d}}$, based on NFW haloes following the differential mass function of Sheth \& Tormen (2002), together with the actual data and best fit of the CARS source redshift distribution, $n\left(z_{\mathrm{s}}\right)$. Right panel: redshift distribution of the expected number density of arcs, $\Gamma_{\mathrm{d}}$, based on the best fit of the sources' redshift distribution. Note that the best fit of the source distribution used in this model overestimates the actual counts, which drop for $z>1.3$ and vanish at $z \approx 2$, thus suppressing the high-redshift tail of $\Gamma_{\mathrm{d}}$. The vertical line marks the redshift limit of the observable sources.
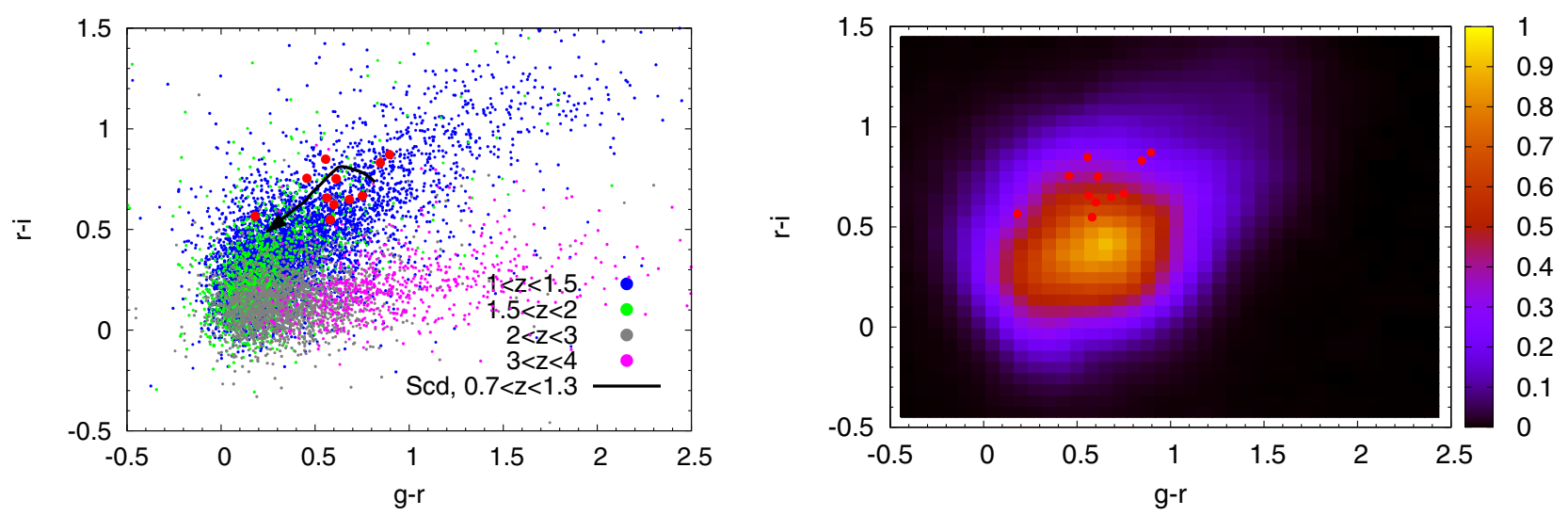

Fig. 5. Left panel: colour-colour diagram for previously known arcs in red and a sub-sample of galaxies observed in the COSMOS field subdivided in redshift bins. The red points denote the arcs previously known in the survey that were used to calibrate the colour selection. The small colour region with arcs shows were the lensed sources lie at redshift $z \approx 1$ (as predicted by our optical depth model) and have well-defined colour properties (as shown by the black arrow referring to a Scd galaxy, for which we derived the photometry out of a synthetic SED; here the arrowhead points in the direction of increasing redshifts). Right panel: same colour-colour diagram, but for all objects detected by the arcfinder.

the actual parameters used to perform the object identification. Here, we highlight the principles that motivate our selection criteria, aiming at the objects with the highest probability to be strongly lensed, which are those at $z \approx 1$. The actual selection is performed by calibrating the method on the colours of known arcs, as we detail in the following section.

\subsection{Observational evidence}

To substantiate our line of argument, we measured photometric properties of the entire sample for all available bands. Magnitudes were measured via aperture photometry

$m=Z P-2.5 \log _{10}(I-S A)$,

where $\mathrm{ZP}$ is the zero-point magnitude (as given in the CARS fits headers), $I$ is the total flux in the aperture given in ADUs, $A$ is the number of pixels within the aperture, and $S=$ 2.5 median-1.5 mean is the background mode (Bertin \& Arnouts 1996) evaluated on the area enclosed within 4.5 arcsec from the outer edge of the aperture. The so-defined background correction accounts for a possible flux contribution of the lens candidate, which should be close to the arc it produces. This implicitly assumes that the lens light-profile decreases linearly in the immediate vicinity of the arc, as justified by its small width. We recall that the aperture of each individual object is defined by the segmentation produced with the arcfinder from the weighted stack of the $g^{\prime}$ and $r^{\prime}$ images (see Sect. 3), and is kept fixed for all bands.

In the left panel of Fig. 5 we plot a colour-colour diagram $(g-r, r-i)$ that show, as red circles, the most evident arcs already known in the literature (More et al. 2012) for which we could derive photometry based on the arcfinder segmentation. The other points refer to a sub-sample of galaxies, grouped into redshift bins, observed with the SUBARU telescope in the COSMOS field (Ilbert et al. 2009). The colour-redshift dependence of the galaxies is clear and shows that lensed sources are indeed associated with galaxies at redshift $z \approx 1$, as expected. The black arrow represents colours of a Scd galaxy, with large spiral arms dominated by a population of young stars for redshifts ranging from $z=0.7$ (start of line) to $z=1.3$ (arrowhead), as derived from a synthetic SED produced by Coleman et al. (1980). This is not used to define the colour cuts, but to show the region where we expect to find galaxies with bright 

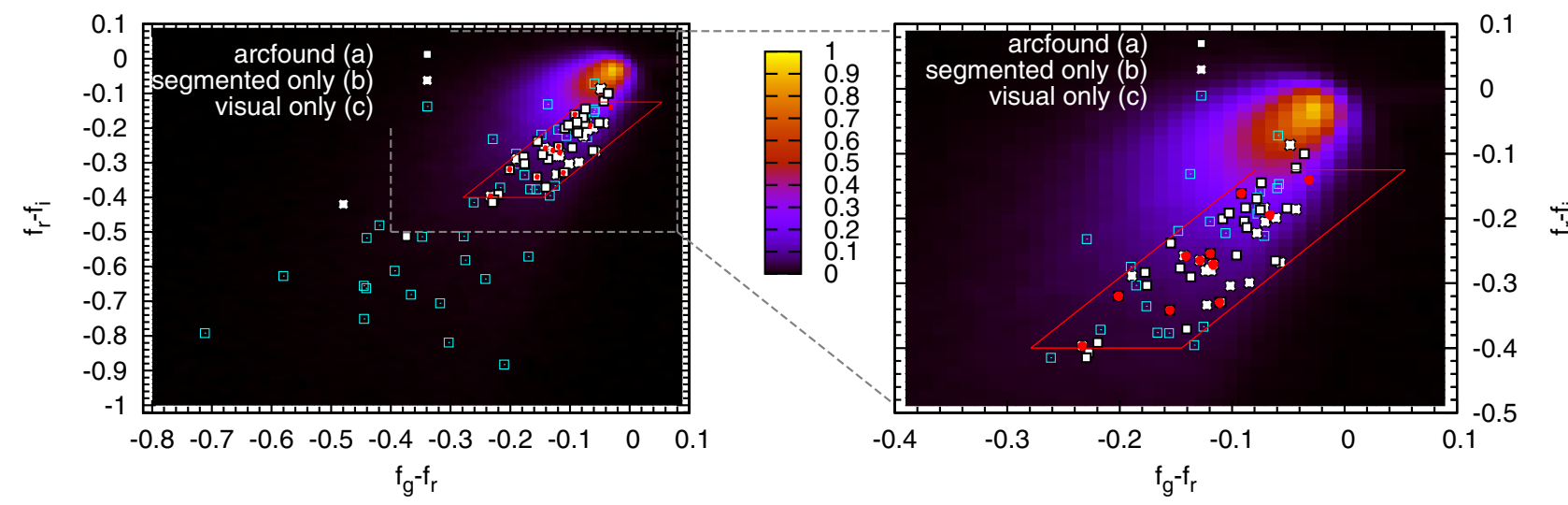

Fig. 6. Flux-related colour-colour space for all arcfinder detections (background intensity), previously known arcs in the field for which we derived the photometry (red points), and region within the red lines that was used to select the final arcs sample. The white squares (a) are the candidates detected by the arcfinder, the white asterisks (b) are those segmented by the arcfinder, but rejected by its geometrical filters, and the cyan void squares (c) are those found purely by visual inspection. The latter are the most difficult sources and are scattered in a very wide area, far from most of the other sources, because of their uncertain photometry.

star-forming regions and therefore well-defined colour properties. In the right panel of Fig. 5 we repeat this exercise, but with all sources detected by the arcfinder. The region covered by the arcs excludes many sources (number density is shown in the background), which helps to drastically reduce the sample contamination. With these arguments, supported by our theoretical model and data alike, we now have a robust basis to help us in distinguishing arcs from other astrophysical sources. We favour this simple colour-colour based method over a full photometric redshift estimate because of its robustness. Small changes in colour might have a strong impact on the photometric redshifts given the difficulty in estimating the light background created by the nearby lenses. In any case, the colour selection is sufficient to constrain the redshift range that has to be investigated.

We can now proceed to define the colour selection, where for convenience we used the flux related quantity

$f_{x}=A^{-1} 10^{0.4\left(K-m_{x}\right)}$,

to express the difference in flux over area for neighbouring bands in the fashion of usual colour-colour plots, instead of the typical definition based on magnitudes. Here, $m_{x}$ is the object magnitude in $x$-band, where $x$ stands for $x=\left[u^{\star}, g^{\prime}, r^{\prime}, i^{\prime}, z^{\prime}\right]$, and $K=26.42$ is the magnitude average of all sources in the $r^{\prime}$-band, resulting in a multiplicative factor introduced for convenience.

The arcs previously known in the survey are shown in Figs. 5 and 6 as red circles. They appear clearly aligned along a relatively narrow region in colour-colour space. These objects were used to define the region marked with red lines in Fig. 6 that we used to select other arc candidates. In the same figure we also plot the objects we identified in the survey as arcs, where details are given in Sect. 6. For the moment, it suffices to say that all objects marked in these plots were not selected according to their colour properties, which makes them an independent check sample. These detections also predominantly populate the same colour-colour space as we used to define the colour-selection, which additionally supports the validity of our approach.

It is difficult to extract detailed statistics from the small sample of known arcs, hence we decided not to use a more sophisticated colour selection criterion. The sample of detections after the colour selection is reduced to 5597 candidates, that is approximately 150 per square degree, versus 970 per square degree in the catalogue obtained with the single-band arcfinder, with comparatively less contamination. This colour selection clearly restricts the detection of arcs to a certain types of lensed sources. Nevertheless, we did not consider this a limitation because (1) we expect the population of lensed sources selected in this way to be the most numerous, ensuring that only a small fraction of arcs is lost, as shown below; (2) sources and lenses are completely uncorrelated because of their large relative separation, which avoids any bias that might be due to physical correlations; and (3) gravitational lensing is a completely achromatic phenomenon independent of the colour of the sources. The giantarcs count, used to infer cosmological information, depends on this colour selection, but this can be easily accounted for just by applying the same colour selection to the expected number density of background sources, which is a trivial task.

\section{Visual validation and catalogue}

The candidates produced with the discussed procedure were finally verified by visually inspecting their colour composite images obtained using the $g^{\prime}, r^{\prime}$ and $i^{\prime}$ bands. We associated a rank to each detection based on three levels:

- 1 = unlikely: arc structures that seem to be physically associated with an astrophysical object or that are located relatively far from a possible lens,

$-2=$ unclear: arc structures with a very promising shape, but still of dubious origin,

- 3 = very likely: a clearly curved arc structure around a possible lens that is its origin, i.e. large ellipticals or significant concentrations of galaxies.

A rank 0 was also used to label artefacts, evident spiral galaxies, or clear noise fluctuations. This simple ranking yields stable results with respect to different observers and even the same observer but at different times. In addition, we inspected the full survey to evaluate the completeness and purity of the sample against a full visual investigation. This very important topic is covered in Sect. 7.

The final catalogue listing all arcs with rank $r \geq 2$ is shown in Tables 2 and 3 with their positions, geometrical properties, photometry, redshifts, and ranks. The first two tables contain the 56 detections that satisfy the colour criterion, while the 34 detections in the third table, most of them found only by visual inspection, do not. The candidates detected by the arcfinder, those that satisfy the tight geometrical constraints, are labelled a. Only 
M. Maturi et al.: Multi-colour detection of gravitational arcs

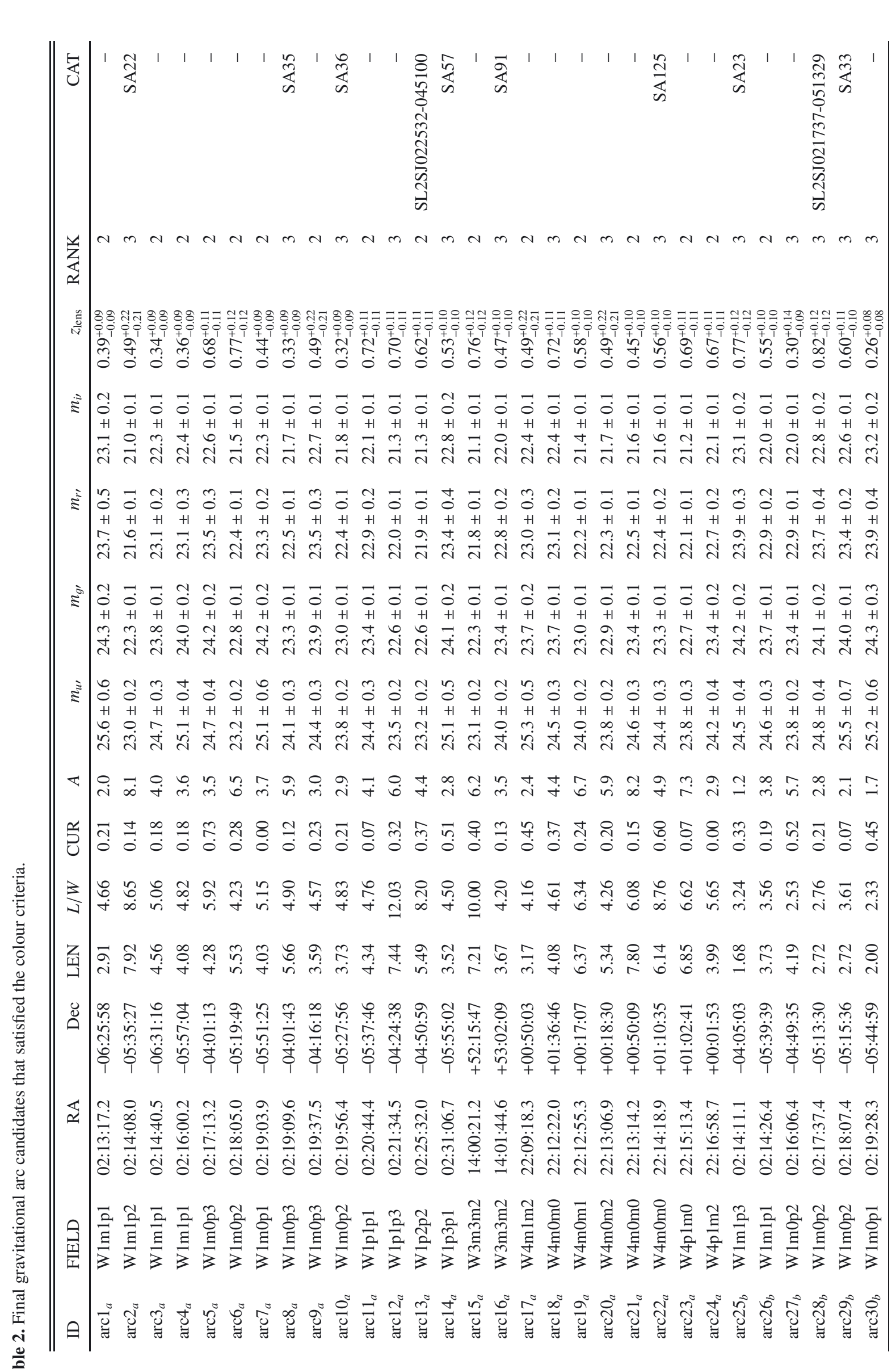

용

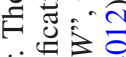

害递完

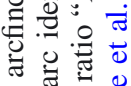

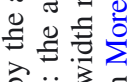

它

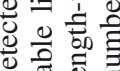

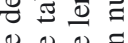

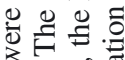

os of

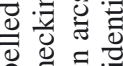

可 0

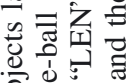

○े

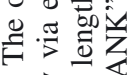

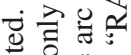

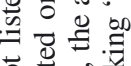

跣

密

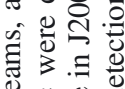

एक

क 흥

후월

so

等

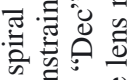

क о

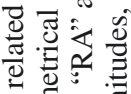

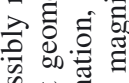

总:

b :

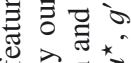

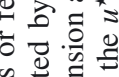

造卷

氞芸

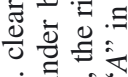

唯

-

VI

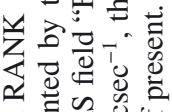

氙 造:

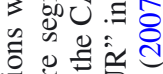

0
0
0
0

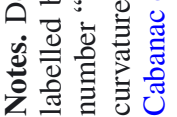

A111, page 7 of 13 


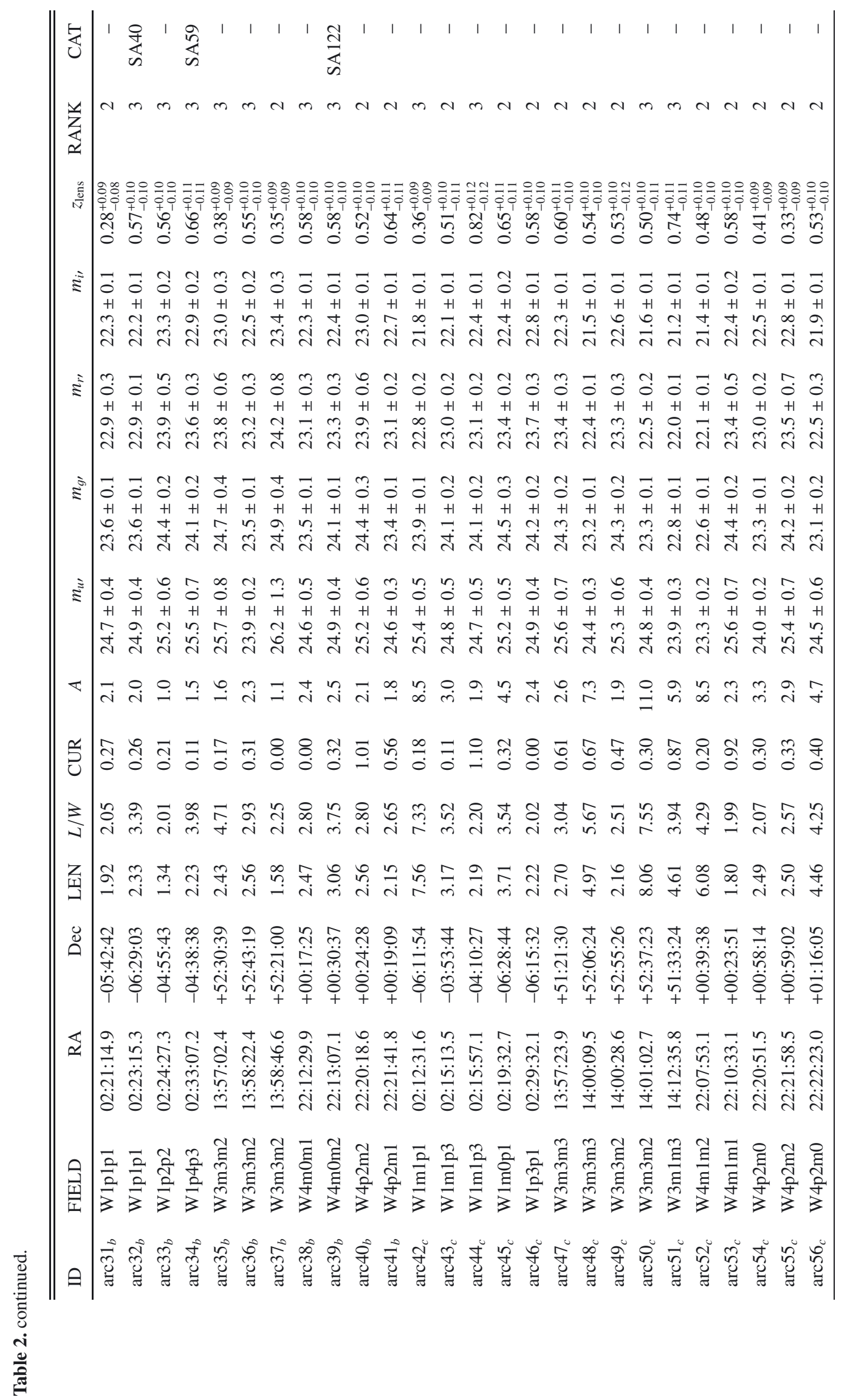




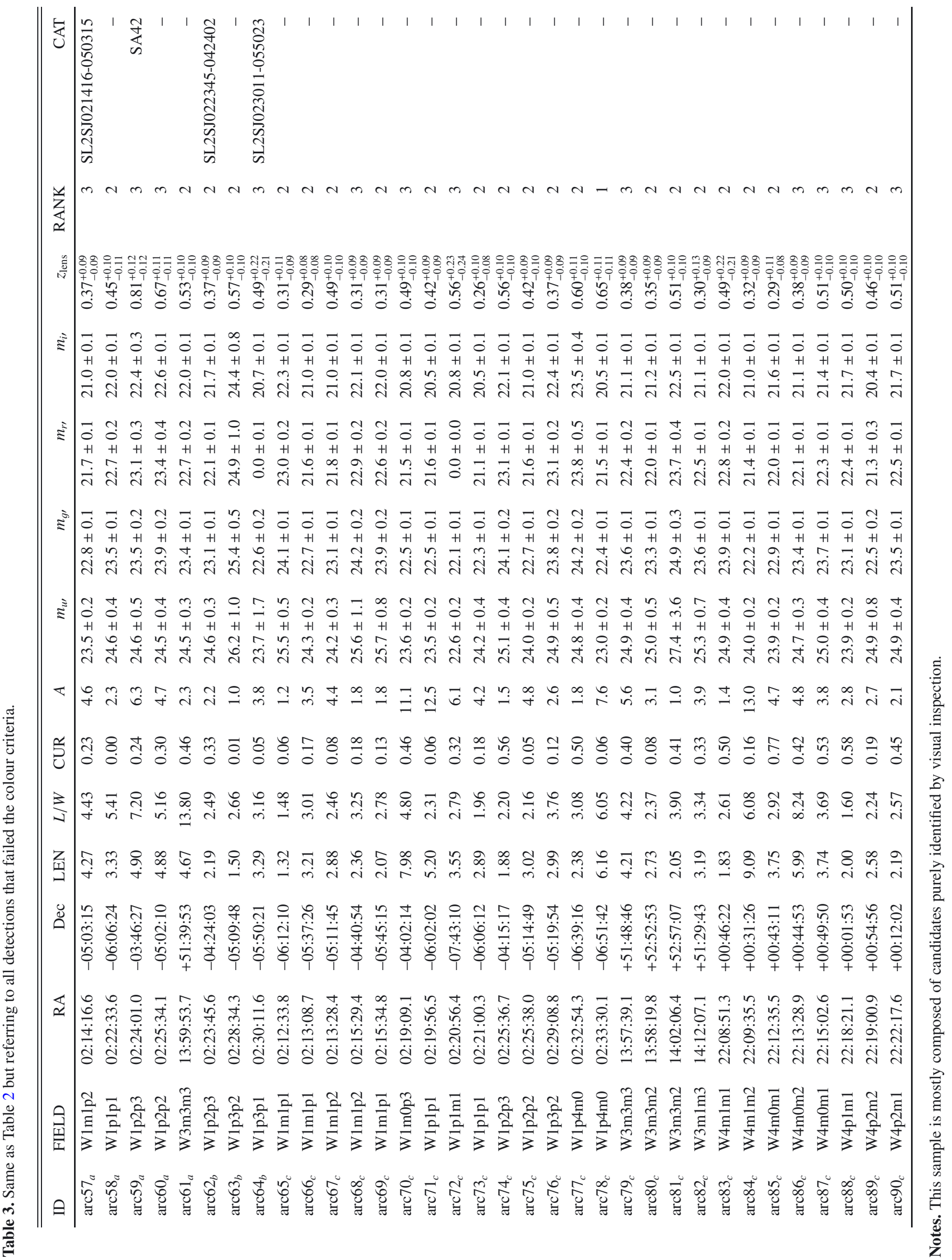


five of them fail the colour criterion. Three of them $\left(\operatorname{arc} 58_{a}\right.$, $\left.\operatorname{arc} 59_{a}, \operatorname{arc} 60_{a}\right)$ are located in the vicinity of very bright stars whose halos might have compromised their photometry, and one ( $\operatorname{arc} 61_{a}$ ) belongs to a complex object of unclear nature that possibly shows multiple arcs. The objects segmented by the arcfinder but discarded by it because they do not fulfil the geometrical constraints are labelled $b$ and all but three of them satisfy the colour constraints. Finally, the candidates detected via checking the whole survey and missed entirely by the arcfinder are labelled c. Most of the visually identified objects fall into category (3) of detections rejected by the colour selection because of their low surface brightness and heavy blending, which makes them generally the most difficult candidates to find. The complete sample consists of 90 objects, 73 of which are newly proposed arc candidates. Postage-stamp cut-outs of their images are available online ${ }^{2}$.

We obtained more candidates than were proposed by More et al. (2012) and Cabanac et al. (2007) not only because of the different arcfinder adopted, but also because of the different selection criteria and because they restricted their sample with respect to the arc-lens separation to $r>14$ arcsec. Note that we dropped two of their candidates, SA 98 and SL2SJ021932-053135, which in our opinion are not lensing features but structures physically associated with the galaxy that is believed to be the lens in the first case, and a spiral arm of a nearby galaxy in the latter. The arcfinder used in this work clearly detected SA 38, but this was rejected because of its high luminosity, which exceeded the maximum we allowed.

\section{Completeness and purity of the colour-selected sample}

We now evaluate the completeness and contamination of the sample, discussed in Sect. 6 for these three candidate subsamples:

a) 29 objects, among 36026 detected by the arcfinder,

b) 20 objects, among 165673 segmented but discarded by the arcfinder's morphological filter,

c) 41 objects only identified with the visual inspection,

and subdivided into those that pass the colour filtering and those that fail it, both within their subsamples and with respect to the total sample. To evaluate the sample completeness, only detections that passed visual inspection were used. In contrast, the complete sample was used to evaluate the contamination level.

The 20 objects segmented by the arcfinder but failing to fulfil all geometrical constraints were the results of the initial calibration run with reduced filtering, not the final detection process. Since they were rejected, counting them as arcfinder detections would be misleading. Since they illustrate the impact of the morphological filter, we still kept them as separate items. The full automatic procedure for the selection of candidates is represented by the 29 objects recognised by the arcfinder and passing all of its filters. While this number might seem to be small, we have to keep in mind that it is similar to the number of arc candidates identified in the CARS fields by previous works and detected with the combined use of arcfinders and visual inspection (e.g. More et al. 2012).

The completeness of the colour selection for the three subsamples and of the total sample is detailed in Table 4. As we show there, the multi-band arcfinder implementing the colour

\footnotetext{
2 http://www.ita.uni-heidelberg.de/ maturi/Public/ arcs
}

Table 4. Completeness of the final arc candidat sample listed in Tables 2, 3, comparing the results obtained before and after the colour selection.

\begin{tabular}{rcccc}
\hline \hline & arcf. (a) & arcf. seg. (b) & visual only (c) & all \\
\hline before col. sel. & 29 & 20 & 41 & 90 \\
after col. sel. & 24 & 17 & 15 & 56 \\
\hline completeness & $83 \%$ & $85 \%$ & $37 \%$ & $62 \%$ \\
\hline
\end{tabular}

Notes. Columns refer to the objects detected by the arcfinder (arcf., labelled a), those segmented but not recognised as arcs by the arcfinder (arcf. seg., labelled b), those identified with the visual inspection only (visual only, labelled $\mathrm{c}$ in the sample catalogue) and the complete sample (all). This shows that the colour selection looses only $17 \%$ of the single-band arcfinder sample (first column). The loss is greater when the visual detections are included, $48 \%$, since many of them have poor photometry because they are blended, faint or fragmented (last column).

selection is complete at a $83 \%$ level with respect to the singleband arcfinder for our sample. Very similar results are obtained for the objects that are segmented but discarded in a further filtering step by the arcfinder because of their geometrical properties: here, the completeness of the colour sample is at a $85 \%$ level. The completeness is lower, at $62 \%$, after including the detections only identified by visual inspection in the complete sample. This is because these detections are faint, fragmented, or heavily blended and therefore have very poor photometry and consequently poorly defined colours. This is clear from Fig. 6, where these objects fall in a colour-colour region devoid of others.

Finally, the contamination level was measured by evaluating the ratio between the number of sources detected by the arcfinder that were not considered to be arcs before (35997 objects) and after (5573 objects) the colour selection. The improvement gained by the use of colour information is evident: a reduction by a factor of 6.5 in the sample contamination. This result largely compensates for the relatively small loss of detections due to multi-band filtering. These values are expected to hold for ground-based observations with similar PSF and depth, but need to be reassessed for space-based surveys. Even if the sample is still heavily contaminated and other criteria have to be included to proceed towards a fully automated method, this strong reduction in the sample contamination is of crucial importance for the present and upcoming surveys, which will cover between $1500 \mathrm{deg}^{2}$ and $15000 \mathrm{deg}^{2}$ in the near future (see e.g. KiDS and Euclid, de Jong et al. 2013; Laureijs et al. 2011), and which can only be handled in an automatic or semi-automatic way.

\section{Conclusions}

The present and upcoming wide-field surveys, such as for the KiDS survey with 1500 square degrees or the ESA Euclid mission with 15000 square degrees of sky coverage, are posing a pressing need for a method automatically detecting stronglensing features in a reliable way. The minimization of human intervention in the detection process is fundamental to avoid a massive use of checking by eye, which is subjective and extremely time consuming. We proposed a detection method and tested it against the CARS data, employing, on one hand, a tailored image segmentation based on coherent patterns in local second-brightness moments, which is capable of isolating elongated objects and of retrieving their length and width, fundamental quantities for characterising and selecting the gravitational arc candidates (Seidel \& Bartelmann 2007). On 
the other hand, a colour-colour based selection, in our case $g^{\prime}-r^{\prime}$ against $r^{\prime}-i^{\prime}$, is motivated by the fact that most of the lensed sources have similar emission properties. We described this behaviour with a model that reproduced the expected redshift distribution of arcs. The model is based on the lensing optical depth expected in a $\Lambda C D M$ cosmology which, in combination with the sources' redshift distribution of the CARS galaxy catalogues, peaks for sources at redshift $z \sim 1$. By furthermore modelling the colours derived from the SED of the galaxies that dominate the population at that redshift, the model well reproduces the colour of the observed arcs. The colour selection of arcs is not a limitation, because the population of the lensed sources selected in this way is the most numerous, ensuring that only a small fraction of arcs is lost. It does not bias measurments derived from gravitational lensing, which is a completely achromatic phenomenon, and the sources are not correlated with the lenses because of their large separation along the line of sight.

To verify the reliability and efficiency of the colour selection we applied our procedure to the CARS data consisting of $37 \mathrm{deg}^{2}$ derived from the $W 1, W 3$, and $W 4$ fields of CFHTLS, and containing $7 \times 10^{6}$ sources (detected with SExtractor). Each step can be summarised as follows:

1. Preprocessing: we created a weighted co-addition of the $g^{\prime}$ and $r^{\prime}$ stacks to produce an image with an enhanced signalto-noise ratio, over which we ran the arcfinder. The $u^{\star}$ and $z^{\prime}$ bands were ignored because of their low signal-to-noise ratio, as well as the $i^{\prime}$ band to avoid blending potentially caused by the lens (probably one or more massive elliptical galaxies) that is in the vicinity of a gravitational arc, which we expect to be more severe in this band.

2. Arcfinder segmentation: we segmented images with the arcfinder to detect all elongated objects and derive their geometrical properties, such as length, length-to-width ratio, and curvature. These geometrical properties were used to select the objects with $L>2.9 \operatorname{arcsec}(15$ pixels $)$ and $L / W_{\min }>4$, and we obtained a sample of 36026 candidates ( $\sim 970$ per square degree).

3. Colour selection: the highest probability of obtaining strongly elongated arcs is given by sources located at redshift $z \approx 1$, where the population is dominated by galaxies with large star-forming regions characterised by relatively uniform colour properties. We therefore selected the sources with respect to their colour-colour $\left(f_{g}-f_{r}, f_{r}-f_{i}\right)$ diagram shown in Fig. 6. This returned a sample of 5597 candidates that were automatically selected.

Note that data acquired by space-based instruments would return results with more depth and, more importantly, purity, not only because of the higher sensitivity but also because of the higher resolution. In fact, because of their very small width gravitational arcs are very sensitive to the image PSF, easily becoming shallower and of smaller $L / W$, which is the most stringent property that distinguishes them from other astrophysical sources.

To validate the candidates we visually inspected their colourcomposite images (based on the $g^{\prime}, r^{\prime}$ and $i^{\prime}$ bands) by removing clear spiral galaxies and image artefacts caused mainly by bright stars and blended sources. We selected only arcs with a curvature compatible with a possible lens such as a large elliptical or an evident over-density of galaxies. This led to the final sample of 49 arc candidates, 20 of which were segmented by the arcfinder, but did not satisfy the geometrical constraints we imposed to define arcs and were therefore not recognised as such by the automatic procedure. In addition, we visually inspected the full survey to evaluate the completeness and purity of the sample retrieved by the arcfinder against such an inspection. In this process, an additional sample of 41 detections, found purely by visual inspection, were included in the final catalogue, which in the end lists 90 objects, 73 of which new proposed arc candidates.

In conclusion, a fully automatic arcfinder is not yet available; we automatically detected 24 objects out of the full sample, but this is mostly due to the limits applied during object segmentation and geometrical filtering. The colour selection we discussed in the paper is very effective in isolating the most promising arc candidates: while completeness is decreased to $83 \%$ with respect to the single-band arcfinder, the sample contamination is drastically reduced by a factor of 6.5. Even if these values are reassessed for different data, they are expected to hold for surveys with similar depth and PSF. This large gain in purity is of crucial importance for the existing and upcoming surveys, which will cover thousands of square degrees that can only be handled in an automatic or semi-automatic way.

Acknowledgements. This work was supported by the Transregional Collaborative Research Centre TRR 33 (MM). S.M. is supported by contract research "Internationale Spitzenforschung II/2-6" of the Baden-Württemberg Stiftung.

\section{Appendix A: Arcfinder segmentation}

We briefly describe the arcfinder method introduced in Seidel \& Bartelmann (2007), the automatic filtering, and post-processing that was added to the basic detection algorithm. This segmentation and first filtering were used for the final colour selection discussed here.

\section{A.1. Introduction}

To measure the orientation of a local source pattern, for example, an area of higher flux inside an ellipse, a simple formalism can be used: the second moments

$Q_{i j} \propto \int_{A}\left(x_{i}-\bar{x}_{i}\right)\left(x_{j}-\bar{x}_{j}\right) I(\boldsymbol{x}) \mathrm{d}^{2} x$

in an area $A$ surrounding the pattern at $\left(\bar{x}_{1}, \bar{x}_{2}\right)$ and - usually reweighed - pixel values $I$ combine into an ellipticity vector $\left(Q_{11}-Q_{22}, 2 Q_{12}\right)^{\mathrm{T}}$ that encloses twice the angle to the $x_{1}$ axis as the pattern itself. For an ellipse, we recover its orientation as long as the area centre is on the major axis. On a constant slope the orientation is parallel to the gradient vector. For an extended source, such as a gravitational lensing arc, we can derive its local orientation when the area is (1) large enough to compensate for noise and (2) small enough to avoid blending with other sources and effects from any arc curvature. Moreover, to recover the orientation directly, the centre pixels must be selected such that they are close to the top of the feature and the integration area is not measuring the slope on both sides.

\section{A.2. Arc detection}

The arcfinder method tests for coherent orientations in areas preferentially centred on features of locally higher intensity. To achieve this, a regular grid of overlapping, disk-shaped areas of equal radii is first placed on the complete image. In three iterations, each area is then displaced to its previous centre of brightness, using a reweighed pixel flux. Areas originally close to a feature move up the slope on both sides, increasing their number density on any significant features' ridge-lines. On a flat or constantly sloped background, areas only move relative to each 
Table A.1. List of the arcfinder parameters.

\begin{tabular}{lll}
\hline \hline Ridge detection & & \\
\hline Gridsize & 7 & Length and width of each cell [pix] \\
Cell spacing & 0.45 & Distance between cells is Cell spacing x Gridsize \\
Threshold cell & 0.45 & Coupling threshold for average coupling over the cell neighbourhood \\
Threshold object & 0.75 & Coupling threshold for inclusion of a single cell into an object \\
Threshold graph & 0.75 & Coupling threshold for graph generation \\
\hline Filtering & & \\
\hline Deblending asymmetry & 30 & Median intensity difference for deblending \\
Deblending distance & 21 & Minimum deblending distance \\
Stars saturation intensity & 500 & Critical intensity above which pixels are considered as saturated \\
Star intensity & 50 & Intensity above which pixels are likely to belong to stars creating diffraction spikes \\
Star-fluxcoeff spike & 3200 & Compute spike radius as fluxcoeff-spike $\times$ flux ${ }^{1 / 2}$ \\
Star-fluxcoeff disk & 420 & Compute disk radius as fluxcoeff-disk $\times$ flux \\
Maxpeakflux & 0.6 & Maximal peak flux in ADUs above the background \\
\hline
\end{tabular}

Notes. For a detailed description we refer to the text.
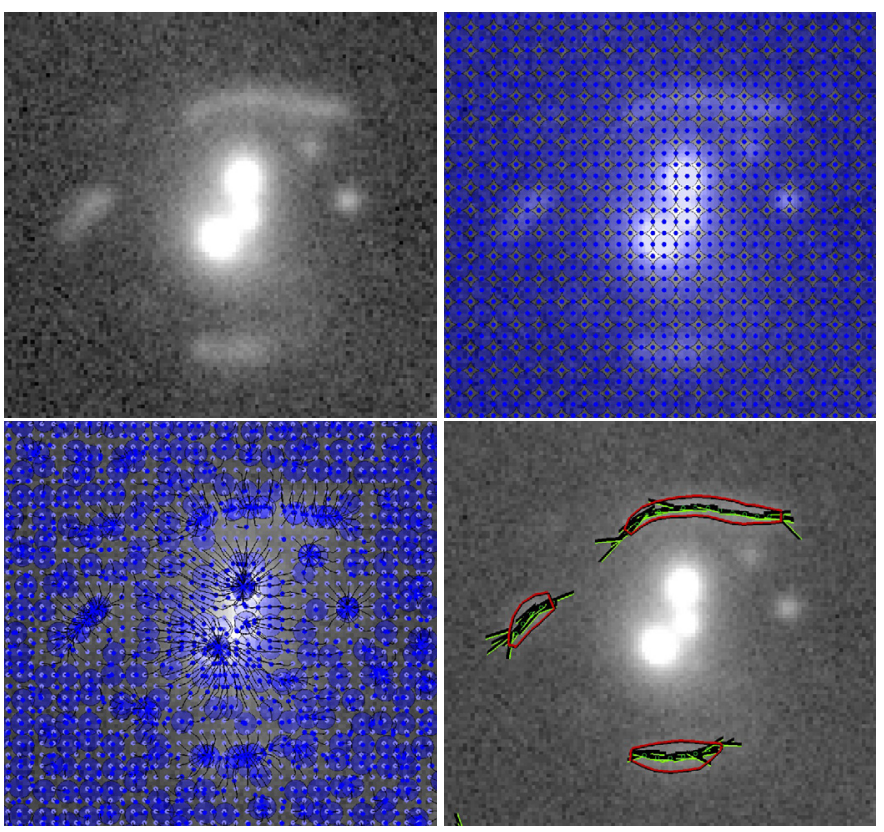

Fig. A.1. Top-left: I-band SL2S J02140-0535 observation, also featuring a giant arc. Top-right: regular grid of disk-shaped areas providing the initial condition of the displacement iterations. Only every second row and column of areas are shown. Bottom-left: areas after three iterations, each moving them to their centre of brightness, and the path they traced. Bottom-right: local orientations for each area, determined using the second-brightness moments. Lines corresponding to coherent areas are highlighted. The red contours show the segmentation of the objects.

other because of pixel noise. At their final positions, each area's orientation is measured using second moments, as described above. To determine coherence, the relative orientations together with the relative placement of areas initially placed close to each other are taken into account. Finally, the centre coordinates of coherent areas are grouped together using a friends-of-friends type algorithm. These groups of coordinates correspond to the initial arcfinder detections.

\section{A.3. Filters and post-processing}

To reduce the number of spurious detections and to expand on the spatial information provided by the area positions and orientations in each detection, filters are applied to the original detections and the shape and flux of each detection are determined. Each of the small detection areas is subjected to a simple fitting procedure that preferentially removes areas centred on point sources and on a background of constant noise without significant structure in the flux distribution. Then, detections with fewer than a minimal amount of valid areas left or shorter than a threshold are removed. Using a fully automatic active contour evolution method (Kass et al. 1988), isophote contours around each detection are determined, which in turn allows for the determination of the flux. Using the flux and a measurement of the background noise in the larger area surrounding each detection, the signal-to-noise ratio is set. A more precise length and a length-to-width ratio is computed from the contour, and minimal thresholds are applied to both photometric and morphological data.

\section{References}

Anguita, T., Barrientos, L. F., Gladders, M. D., et al. 2012, ApJ, 748, 129 Bartelmann, M. 2010, Class. Quant. Grav., 27, 233001

Bartelmann, M., Steinmetz, M., \& Weiss, A. 1995, A\&A, 297, 1

Bartelmann, M., Huss, A., Colberg, J. M., Jenkins, A., \& Pearce, F. R. 1998, A\&A, 330, 1

Bayliss, M. B., Hennawi, J. F., Gladders, M. D., et al. 2011, ApJS, 193, 8

Benjamin, J., Heymans, C., Semboloni, E., et al. 2007, MNRAS, 381, 702

Bertin, E., \& Arnouts, S. 1996, A\&AS, 117, 393

Bolton, A. S., Burles, S., Koopmans, L. V. E., Treu, T., \& Moustakas, L. A. 2006, ApJ, 638, 703

Boulade, O., Charlot, X., Abbon, P., et al. 2003, in Proc. SPIE 4841, eds. M. Iye, \& A. F. M. Moorwood, 72

Bradač, M., Vanzella, E., Hall, N., et al. 2012, ApJ, 755, L7

Broadhurst, T., Benítez, N., Coe, D., et al. 2005, ApJ, 621, 53

Cabanac, R. A., Alard, C., Dantel-Fort, M., et al. 2007, A\&A, 461, 813

Clowe, D., Gonzalez, A., \& Markevitch, M. 2004, ApJ, 604, 596

Coe, D., Zitrin, A., Carrasco, M., et al. 2013, ApJ, 762, 32

Coleman, G. D., Wu, C.-C., \& Weedman, D. W. 1980, ApJS, 43, 393

Coles, J. 2008, ApJ, 679, 17

Dalal, N., Holder, G., \& Hennawi, J. F. 2004, ApJ, 609, 50

de Jong, J. T. A., Verdoes Kleijn, G. A., Kuijken, K. H., \& Valentijn, E. A. 2013, Exp. Astron., 35, 25

Erben, T., Schirmer, M., Dietrich, J. P., et al. 2005, Astron. Nachr., 326, 432

Erben, T., Hildebrandt, H., Lerchster, M., et al. 2009, A\&A, 493, 1197

Fassnacht, C. D., Moustakas, L. A., Casertano, S., et al. 2004, ApJ, 600, L155

Faure, C., Kneib, J.-P., Covone, G., et al. 2008, ApJS, 176, 19

Fedeli, C., Meneghetti, M., Bartelmann, M., Dolag, K., \& Moscardini, L. 2006, A\&A, 447, 419

Gavazzi, R., Marshall, P. J., Treu, T., \& Sonnenfeld, A. 2014, ApJ, 785, 144

Horesh, A., Ofek, E. O., Maoz, D., et al. 2005, ApJ, 633, 768 
M. Maturi et al.: Multi-colour detection of gravitational arcs

Ilbert, O., Capak, P., Salvato, M., et al. 2009, ApJ, 690, 1236

Jackson, N. 2008, MNRAS, 389, 1311

Joseph, R., Courbin, F., Metcalf, R. B., et al. 2014 [arXiv: 1403. 1063]

Kass, M., Witkin, A., \& Terzopoulos, D. 1988, Int. J. Comput. Vis., 1, 321

Kneib, J.-P., Ellis, R. S., Santos, M. R., \& Richard, J. 2004, ApJ, 607, 697

Kochanek, C. S. 2006, in Saas-Fee Advanced Course 33: Gravitational Lensing:

Strong, Weak and Micro, eds. G. Meylan, P. Jetzer, \& P. North (Berlin: Springer), 91

Laureijs, R., Amiaux, J., Arduini, S., et al. 2011 [arXiv: 1110. 3193]

Li, G.-L., Mao, S., Jing, Y. P., et al. 2005, ApJ, 635, 795

Limousin, M., Cabanac, R., Gavazzi, R., et al. 2009, A\&A, 502, 445

Meneghetti, M., Bolzonella, M., Bartelmann, M., Moscardini, L., \& Tormen, G. 2000, MNRAS, 314, 338

Meneghetti, M., Bartelmann, M., \& Moscardini, L. 2003, MNRAS, 340, 105

Meneghetti, M., Bartelmann, M., Dahle, H., \& Limousin, M. 2013, Space Sci. Rev., 177, 31

Merten, J., Cacciato, M., Meneghetti, M., Mignone, C., \& Bartelmann, M. 2009, A\&A, 500, 681

Merten, J., Coe, D., Dupke, R., et al. 2011, MNRAS, 417, 333
More, A., Cabanac, R., More, S., et al. 2012, ApJ, 749, 38

Navarro, J. F., Frenk, C. S., \& White, S. D. M. 1997, ApJ, 490, 493

Oguri, M., Bayliss, M. B., Dahle, H., et al. 2012, MNRAS, 420, 3213

Richard, J., Stark, D. P., Ellis, R. S., et al. 2008, ApJ, 685, 705

Richard, J., Kneib, J.-P., Ebeling, H., et al. 2011, MNRAS, 414, L31

Schneider, P., Ehlers, J., \& Falco, E. E. 1999, Gravitational Lenses, Astron. Astrophys. Lib. (Springer)

Seidel, G., \& Bartelmann, M. 2007, A\&A, 472, 341

Sheth, R. K., \& Tormen, G. 2002, MNRAS, 329, 61

Suyu, S. H., Marshall, P. J., Auger, M. W., et al. 2010, ApJ, 711, 201

Tewes, M., Courbin, F., Meylan, G., et al. 2013, A\&A, 556, A22

Torri, E., Meneghetti, M., Bartelmann, M., et al. 2004, MNRAS, 349, 476

Verdugo, T., Motta, V., Muñoz, R. P., et al. 2011, A\&A, 527, A124

Wambsganss, J. 1998, Liv. Rev. Rel., 1, 12

Willmer, C. N. A., Faber, S. M., Koo, D. C., et al. 2006, ApJ, 647, 853

Zacharias, N., Finch, C., Girard, T., et al. 2010, AJ, 139, 2184

Zitrin, A., \& Broadhurst, T. 2009, ApJ, 703, L132

Zitrin, A., Broadhurst, T., Umetsu, K., et al. 2009, MNRAS, 396, 1985 